\title{
Measurement and prediction for air flow drag in different packing materials
}

\author{
C. Rautenbach ${ }^{1}$, B. M. Halvorsen ${ }^{2,3}$, E. du Plessis ${ }^{1}$, S. Woudberg ${ }^{1}$ \\ \& J. P. du Plessis ${ }^{1}$ \\ ${ }^{1}$ Department of Mathematical Sciences, Applied Mathematics Division, \\ Stellenbosch University, South Africa \\ ${ }^{2}$ Institute for Process, Energy and Environmental Technology, \\ Telemark University College, Norway \\ ${ }^{3}$ Telemark Technological R\&D Centre (Tel-Tek), Norway
}

\begin{abstract}
Packed bed reactors are widely used in industry to improve the total contact area between two substances in a multiphase process. In some cases, like for the packing elements of some $\mathrm{CO}_{2}$ absorption towers, the packing material can be of such geometric nature that during discharge through them different flow conditions can be present in different parts of the packing. This renders prediction of pressure drops quite difficult. This paper concerns experimental and modelling activities to improve predictive equations for pressure drops over a packed bed of Raschig rings. It is shown that the application of some corrective measures can dramatically improve the correlation between theory and experiment, but that more research is needed in this field, regarding both carefully controlled experiments and mathematical modelling.

Keywords: pressure drop, porous media, packing materials, Raschig rings, drag models.
\end{abstract}

\section{Introduction}

In the chemical engineering industry packed bed reactors are widely used to improve the total contact area between two substances in a multiphase process. The process typically involves forced convection of liquid or gas through either structured or dumped solid packings. Applications of such multiphase processes include mass 


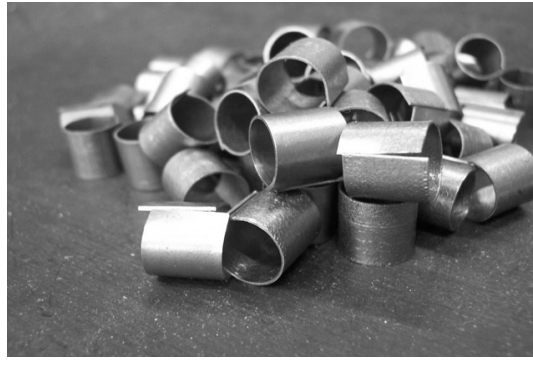

(a)

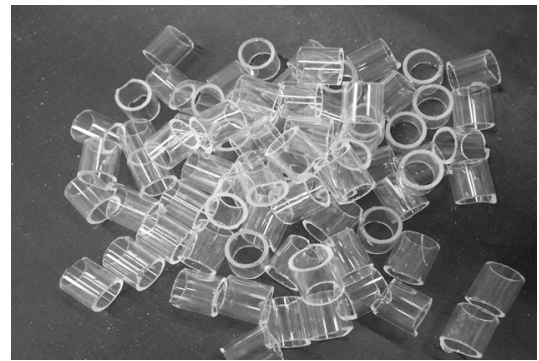

(b)

Figure 1: Typical examples of random packings. (a) Metallic Raschig rings and (b) glass Raschig rings.

transfer to catalyst particles forming the packed bed and the adsorption of gases or liquids on the solid packing.

For reactor design the drag laws are needed to predict the pressure drops over such reactors. In many cases the packing material can be of such geometric nature that during discharge through them different flow conditions can be present in different parts of the packing. This renders prediction of pressure drops quite difficult. This paper concerns experimental and modelling activities to improve predictive equations for pressure drops over a packed bed of Raschig rings.

An experimental study on the determination of air flow pressure drops over different packing materials was carried out at the Telemark University College in Porsgrunn, Norway. The packed bed consisted of a cylindrical column of diameter $0.072 \mathrm{~m}$ and height $1.5 \mathrm{~m}$, filled with different packing materials. Air was pumped vertically upwards through a porous distributor to allow for a uniform inlet pressure. Resulting pressure values were measured at regular height intervals within the bed. Due to the geometric nature of a Raschig ring packing the wall effects, namely the combined effects of extra wall shear stress due to the column surface and channelling due to packing alignment adjacent to a solid column surface, were assumed to be negligible. Several mathematical drag models exist for packed beds of granular particles and an important question arises as to whether they can be generalized in a scientific manner to enhance the accuracy of predicting the drag for different kinds of packing materials. Problems with the frequently used Ergun equation, which is based on a tubular model for flow between granules and then being empirically adjusted, will be discussed. Some theoretical models that improve on the Ergun equation and their correlation with experimental work will be discussed. It is shown that a particular pore-scale model, that allows for different geometries and porosities, is superior to the Ergun equation in its predictions. Also important in the advanced models is the fact that it could take into account anomalies such as dead zones where no fluid transport is present and surfaces that do neither contribute to shear stress nor to interstitial form drag. The overall conclusion is that proper modelling of the dynamical situation present in the packing 


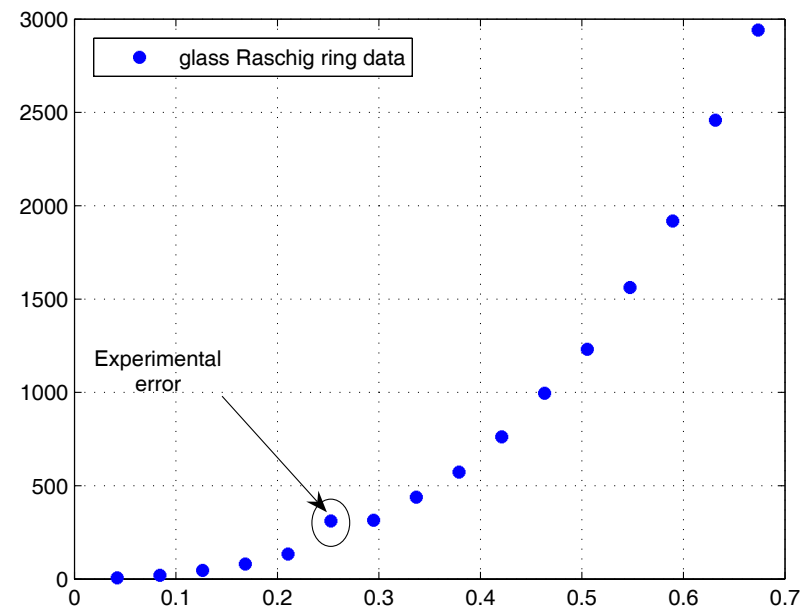

Figure 2: Experimental results for glass Raschig rings.

can provide drag models that can be used with confidence in a variety of packed bed applications.

There is a wide range of different packing materials available. The packing material used varies from application to application. Factors that need to be considered include the pressure drop produced by the packed bed, chemical stability of the packing and size of the packing, to name but a few. Porous media created by using the packing materials illustrated in Figure 1 are called random dumped packings, as they are randomly placed into the container. Raschig rings and small glass spheres were provided by the TUC in Porsgrunn Norway and were used to produce the experimental results presented in this study.

An example of data retrieved during the experiments conducted at the TUC is given in Figure 2. As indicated in Figure 2, there is a data point that does not follow the trend of the rest of the data. This is assumed to be caused by experimental error and is ignored for the remainder of the analysis.

\section{Existing models used to predict flow behavior through porous packed beds}

In this section some existing available models are discussed. For convenience of comparison all models will be rewritten in terms of a $F$, defined by

$$
\frac{\Delta p}{L}=\mu q F
$$


with $\Delta p$ the measured pressure drop, corrected for gravity, and $L$ the bed height. The Reynolds number, $R e_{p}$, is defined as:

$$
R e_{D_{p}} \equiv \frac{\rho q D_{p}}{\mu}
$$

for use in the present study.

\subsection{Ergun equation}

The Ergun equation [1], empirically based on results obtained from experimental packed beds of identical spheres, is given in the present notation, with $D_{p}$ the particle diameter, as:

$$
F D_{p}^{2}=\frac{150(1-\epsilon)^{2}}{\epsilon^{3}}+\frac{1.75(1-\epsilon)}{\epsilon^{3}} \cdot R e_{D_{p}}
$$

The empirically based constants compensate for the assumptions made in the capillary model. One assumption is that the porous medium is statistically uniform so that there is no channelling. Of course this is a crude assumption as channelling is common place in practical applications. Another more practical assumption is that the column diameter is large in comparison to the particle dimensions. The Ergun equation [1] also assumes a uniform particle size. As more data on irregular particles becomes available, the modelling can be improved to represent a wider spectrum of packed bed geometries.

\subsection{RUC model}

An RUC drag model, [2], is used in this work to give a possible prediction of single phase flow through the packing elements of a $\mathrm{CO}_{2}$ absorption tower. This universal model can be applied to different types of porous media and for this work our interest is in the granular and foam versions.

\subsubsection{Granular RUC model}

The granular RUC model is model aims to approximate porous media such as sand, consisting of small granular particles. The expression for the drag factor for granular porous media is expressed as:

$$
F D_{p}^{2}=\frac{25.4(1-\epsilon)^{4 / 3}}{\left(1-(1-\epsilon)^{1 / 3}\right)\left(1-(1-\epsilon)^{2 / 3}\right)^{2}}+\frac{c_{d}(1-\epsilon)}{2 \epsilon\left(1-(1-\epsilon)^{2 / 3}\right)^{2}} R e_{D_{p}}
$$

The form drag coefficient, $c_{d}$, should typically be determined either numerically or empirically and is frequently assigned the value 1.9. The pressure drop can then be determined via the drag factor $F$ from equation (4). 


\subsubsection{Foam model}

The RUC foam model was developed to accurately predict flow behavior through foamlike porous media [3]. A typical example is a spongelike metallic foams. The two variations that exist in the foam model are the doubly staggered model and the singly staggered model, the latter yielding a smaller pressure drop for the same discharge. The drag factor is given as:

$$
F d^{2}=\frac{24 \psi^{2}(\psi-1)}{\epsilon^{2}}+\frac{c_{d} \psi^{2}(\psi-1)}{2 \epsilon^{2}(3-\psi)} \cdot \frac{\rho q d}{\mu},
$$

in the case of the doubly staggered model and as:

$$
F d^{2}=\frac{36 \psi^{2}(\psi-1)}{\epsilon^{2}}+\frac{c_{d} \psi^{2}(\psi-1)}{\epsilon^{3}(3-\psi)} \cdot \frac{\rho q d}{\mu},
$$

for the singly staggered model. An expression for the geometric factor, $\psi$, as given in equations (5) and (6), is given as:

$$
\psi=2+2 \cos \left[\frac{4 \pi}{3}+\frac{1}{3} \cos ^{-1}(2 \epsilon-1)\right]
$$

for foamlike media. The micro-scale parameter $d$ is given by the length of a cube that would produce $N$ cubes in the total packing volume. The total number of particles in a fixed bed is represented by $N$.

\subsection{The Sonntag correction}

A small change in the porosity has a large impact on the pressure drop and thus this effect can have a large influence on the pressure drop, predicted by the models. Sonntag [4] introduced the influence of the fraction $m$ of the inner volume $V_{i}$ of a Raschig ring that is stagnant and does not contribute to the shear stress nor to the interstitial form drag. After experimental correlations he stated that only approximately $20 \%$ of the inner volume of the ring is available for flow, i.e. $m=$ 0.2 . The effect of the decrease in the volume available for flow is a decrease in the effective porosity.

\subsection{Nemec's equation}

Nemec [4] applied the Sonntag correction to the Ergun equation. In Nemec's work the experimentally determined values for the tortuosity, $\chi$, and the friction factor, $f$, are kept the same as stated by Ergun. The reasoning is that when Sonntag derived his $20 \%$ criterion he used the original Ergun equation. Thus if adapted values for the tortuosity, $\chi$, and the friction factor, $f$, are used, say for a bed consisting 
of equivalent solid cylinders, the value of Sonntag's correction would change. The equation put forward by Nemec can be written as:

$$
\begin{gathered}
F d_{e}^{2}=\frac{150(1-\epsilon)^{2}}{\epsilon^{3}}\left[\frac{\epsilon^{3}}{\left(1-(1-\epsilon)\left(V_{f c}-m V_{i}\right) / V_{p}\right)^{3}}\right] \times\left[\frac{d_{e}\left(S_{f c}+m S_{i}\right)}{6 V_{p}}\right] \\
+\frac{1.75 d_{e} \rho q(1-\epsilon)}{\mu \epsilon^{3}}\left[\frac{\epsilon^{3}}{\left(1-(1-\epsilon)\left(V_{f c}-m V_{i}\right) / V_{p}\right)^{3}}\right] \\
\times\left[\frac{d_{e}\left(S_{f c}+m S_{i}\right)}{6 V_{p}}\right]^{2},
\end{gathered}
$$

with $V_{i}$ the volume of the inner void cylinder, $V_{p}$ the particle volume and $V_{f c}$ the volume of a hypothetical full cylinder with the same outer dimensions. In equation (8) the surfaces are indicated by an $S$ and the subscripts have the same meaning as previously mentioned volumes. The fraction of the inner void of each ring available for flow is denoted by $m$ and can be taken as $20 \%$ according to Sonntag [4]. The equivalent particle diameter is defined as $6 V_{p} / S_{p}$ and is denoted by $d_{e}$ in equation (8).

\subsection{Mackowiac's equation}

Using experimental results from Raschig ring packings, Mackowiak [5] arrived at the following drag equation for perforated Raschig ring packings when he investigated the influence of the fluid-solid interface on the drag:

$$
F=\frac{1}{\mu q}\left(\frac{725.6}{R e_{v}}+3.203\right)(1-\varphi)\left(\frac{1-\epsilon}{\epsilon^{3}}\right)\left(\frac{F_{v}^{2}}{d_{p} K}\right) .
$$

Here the form factor $\varphi$ becomes zero for non-perforated packings, like Raschig rings.

According to Mackowiak the value for the resistance coefficient, $\psi_{0}$, has been determined experimentally and has been found to be given by:

$$
\psi_{0}=\left(\frac{725.6}{R e_{v}}+3.203\right),
$$

with $R e_{v}$ the modified Reynolds number and is defined as:

$$
R e_{v}=\frac{q d_{p}}{(1-\epsilon) \mu} K
$$

\section{Comparative results}

The comparisons of the models to the experimental data for glass Raschig rings are given in Figure 3. The models were compared to the data and to each other, for metallic Raschig rings in Figure 4 (a). The metallic rings produced a higher 


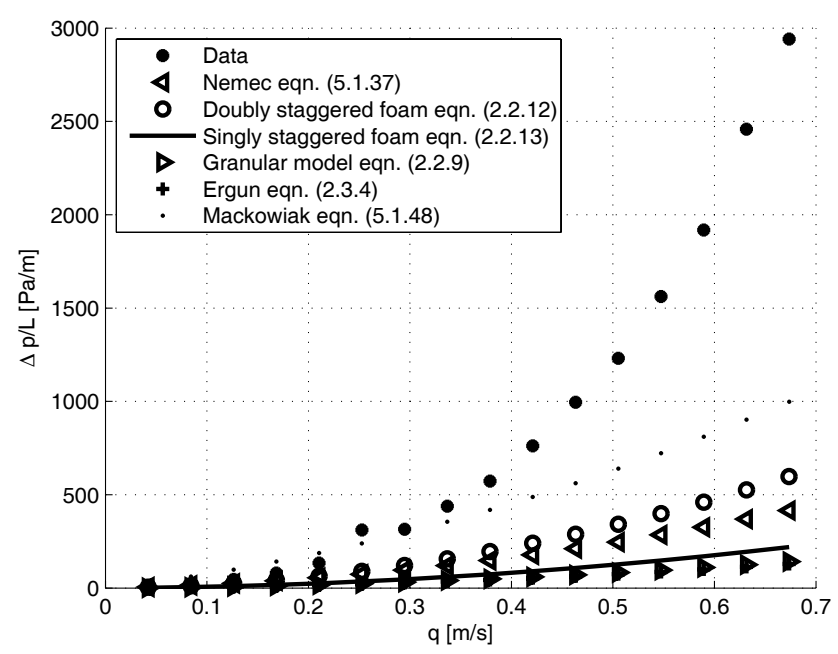

Figure 3: Comparison of model with the data for glass Raschig rings (refer to Figure 1(a)).

porosity than the glass ring bed. The result is that the effect of the wall on the over all pressure drop is lower with the metallic rings. This is also slightly evident from Figures 3 and 4 (a). It is suspected that wall effects are the cause of the difference in curvature of the models and the actual data. Confirmation of this suspicion is that when pressure drop measurements through small spherical particles were collected, there were no curvature discrepancy. In Figure 4 (b) the comparison of the Granular RUC model to the data acquired with flow through non-uniform spherical particle is given. Thus the difference in curvature could not be caused by incorrect data processing or incorrect experimental methods.

\section{Adaptations}

\subsection{Shape-factor}

In the case of irregular shaped packings, shape factors can be used to determine the equivalent diameter of a sphere with the same volume as the element or particle (nominal diameter). The sphericity, $\phi_{s}$, of an element is the ratio of the surface of the equivalent sphere to the actual surface area of the element. In Figure 5 the effect of a shape factor (sphericity) is investigated. It is evident that the sphericity marks a significant improvement on the RUC granular model and Ergun equation's accuracy. 


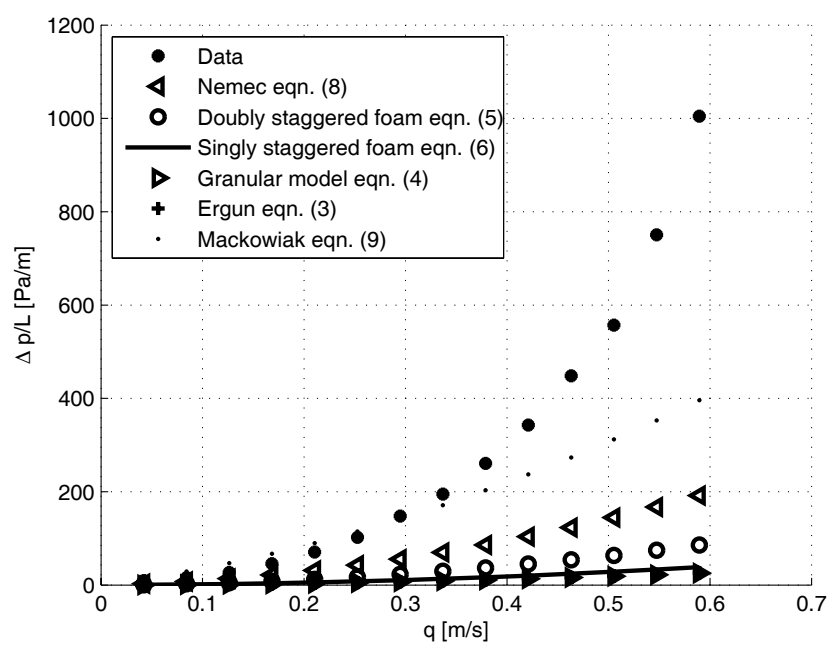

(a)

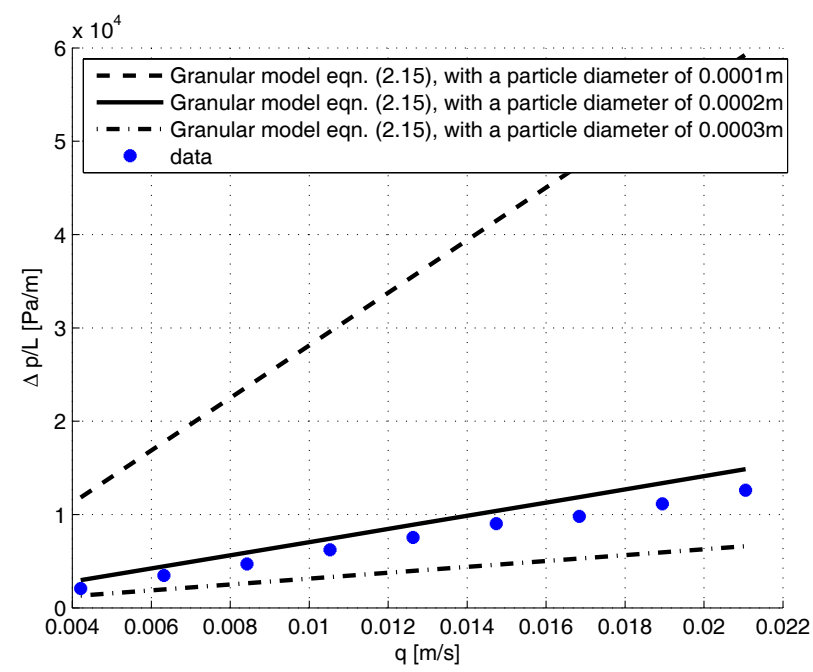

(b)

Figure 4: (a) Comparison of model with the data for metallic Raschig rings Figure 1 (b). (b) Comparison of the RUC granular model [2] with experimental data given a range of particle sizes. $100-200 \mu \mathrm{m}$ powder was used to acquire the data. 


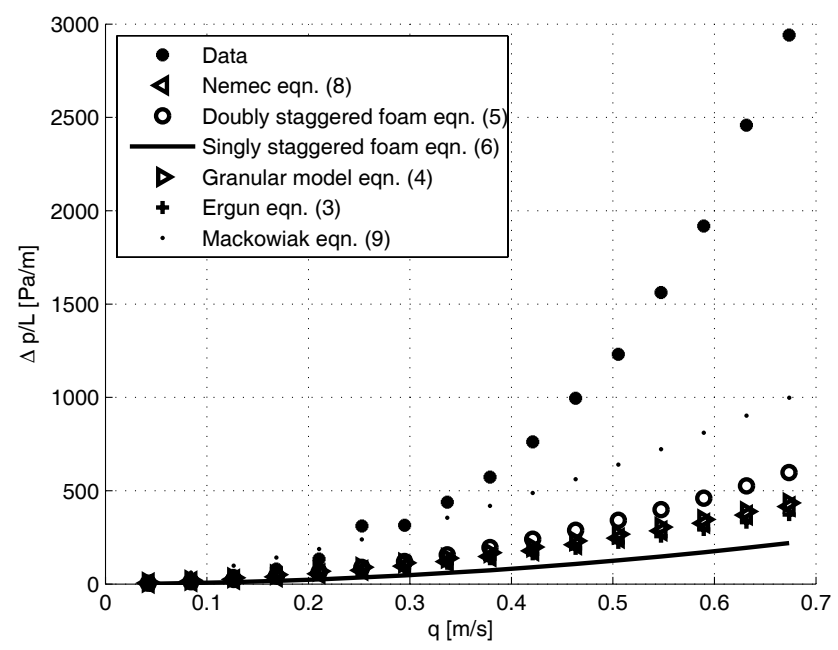

Figure 5: Investigation of the effect of the sphericity on the Ergun equation and the RUC granular model.

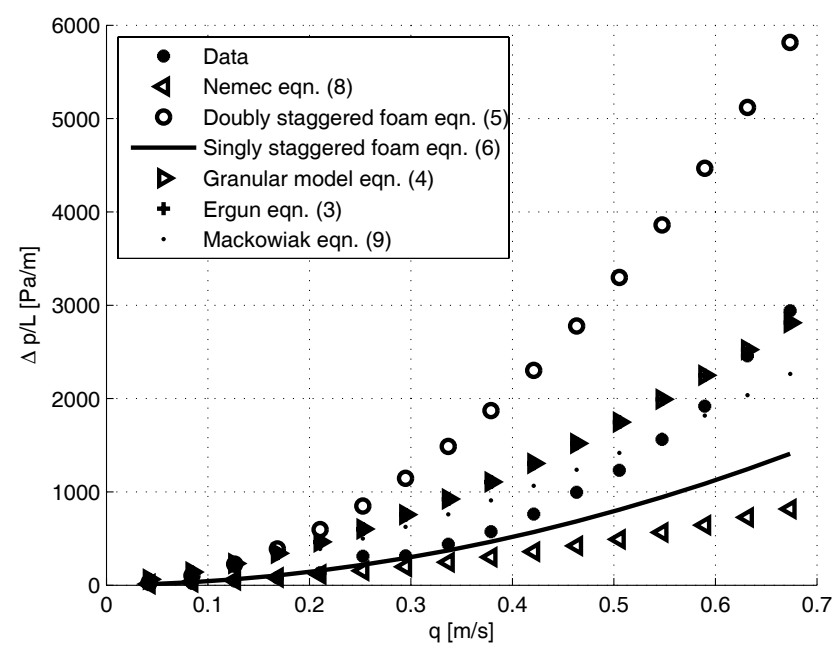

Figure 6: (a) Comparison of models with the Sonntag correction equal to $20 \%$ (with glass Raschig rings). (b) Pressure gradients of the different models and the data. 


\subsection{The Sonntag correction}

This stagnant region within the rings can also be the reason for the under prediction of the Ergun equation. In Figure 6 the effect of the Sonntag correction is given. Thus using the sphericity and the Sonntag correction the best correlation to the experimental data is obtained.

\section{Conclusion}

The major contribution of this work was the generation of a set of data by means of experiments and analyses of possible predictive models. They produced satisfactory correlations to data and thus provide confidence in the capability of mathematical models to predict experimental trends for various fixed bed reactors.

The initial aim of using the spherical particle powders in the experimental part of this study was to check how experimental results would compare with the well known Ergun equation. As the Ergun equation was adapted empirically, based on the data obtained for flow through uniform spheres, it is expected to describe the flow through non-uniform spheres to some extent. The reason for such an assessment was because of the non-linearity of the data acquired with Raschig rings. The curvature differed from what the models predicted and the suspicion arose that the data may have been processed incorrectly. Thus, after the powders produced data that had the same behavior as the models, especially the Ergun equation, it can be concluded that the problem with the Raschig rings was not the result of incorrect data processing. The main cause is expected to be wall effects, due to the column to ring size ratio being small, only about 10 . If this ratio is much larger, i.e. if $d_{c} / d_{p}>>10$, the effect of the wall can be ignored [5].

With the incorporation of the Sonntag correction all the models perform better. On the modelling side the Sonntag correction was applied with great success to existing empirical and pore-scale models. Using any of the models discussed in this work without Sonntag's correction will result in a under-prediction of the pressure drop for Raschig ring experiments.

The deviations in the models from the data could be attributed to a variety of different effects. Almost none of these effects could be pin-pointed satisfactorily in this work due to time constraints. It is thus advisable to verify which effects predominated in both the Raschig ring and powder beds. The next logical step would then also be to find ways in which to combat the effects or to adapt the models to take these effects into account.

\section{References}

[1] Ergun, S., Fluid flow through packed columns. Chemical Engineering Progress, 48, pp. 89-94, 1952.

[2] du Plessis, J.P. \& Woudberg, S., Pore-scale derivation of the ergun equation to enhance its adaptability and generalization. Chem Eng Sci, 63, pp. 2676-2586, 2008. 
[3] Crosnier, S., du Plessis, J.P., Riva, R. \& Legrand, J., Modeling of gas flow through isotropic metallic foams. Journal of Porous Media, 9, pp. 35-54, 2006.

[4] Nemec, D. \& Levec, J., Flow through packed bed reactors: 1. single-phase flow. Chemical Engineering Science, 60, pp. 6947-6957, 2005.

[5] Mackowiak, J., Extended channel model for prediction of the pressure drop in single-phase flow in packed columns. Chemical Engineering Research and Design, 2008.

[6] Mcdonald, I.F., El Sayed, M.S., Mow, K. \& Dullien, F.A.L., Flow through porous media - the ergun equation revisited. Ind Eng Chem Fundam, 18, pp. 199-208, 1979. 